\title{
Saturación pre y postductal en recién nacidos sanos de la Ciudad de México
}

\author{
Pre- and post-ductal oxygen saturation among healthy \\ newborn babies from Mexico City
}

\author{
Corinne Michelle Lozano Duau, * Rodrigo Hernández Benítez, \\ José Iglesias Leboreiro, ${ }^{\S}$ Isabel Bernárdez Zapata, "I Desireé Vidaña Pérez ${ }^{\Uparrow}$
}

\section{Resumen}

Objetivo: Describir la saturación pre y postductal realizada mediante el tamiz cardiaco recomendado por la American Academy of Pediatrics (AAPde todos los recién nacidos ingresados al cunero fisiológico, a una altitud de 2,640 metros sobre el nivel del mar (Cuidad de México). Material y métodos: Estudio transversal, se llevó a cabo durante el periodo de marzo a agosto de 2018 , todos los tamices se realizaron posterior a las 24 horas de vida, utilizamos el algoritmo recomendado por la AAP. La toma de oximetría se realizó en dos sitios: mano derecha (preductal) y cualquiera de los pies (postductal). Se incluyeron 292 recién nacidos (RN), de los cuales 256 fueron $\mathrm{RN}$ de término, y $36 \mathrm{RN}$ de pretérmino tardío. Resultados: $\mathrm{La}$ media de saturación preductal fue de 95.5\% (IC 95\% 95.2-95.8) y postductal de $95.8 \%$ (IC 95\% 95.5-96.1). Conclusiones: Se establecieron valores para recién nacidos a la altitud de la Cuidad de México, con los cuales podemos sugerir la aplicación de forma segura del tamiz cardiaco con los lineamientos de la AAP en nuestro medio.

Palabras clave: Saturación en recién nacido sano, altitud de Cuidad de México, tamiz cardiaco.

\section{INTRODUCCIÓN}

Las cardiopatías congénitas (CC) representan aproximadamente $1 \%$ de todos los desórdenes congénitos al naci-

\section{Abstract}

Objective: To describe the pre and post ductal saturation performed by the cardiac screen recommended by the American Academy of Pediatrics (AAP) of all newborns admitted to the physiological nursery, at a height of 2,640 meters above sea level (Mexico City). Material and methods: A cross-sectional study conducted during the period from March to August of 2018, all the sieves were performed after 24 hours of life, we use the algorithm recommended by the AAP. Oximetry was taken at two sites: right hand (preductal) and any of the feet (postductal). We included 292 newborns $(\mathrm{RN})$, of which 256 were term RN, and 36 late preterm RN. Results: Mean preductal saturation was $95.5 \%$ (95\% Cl 95.295.8) and $95.8 \%$ postductal ( $95 \% \mathrm{Cl} 95.5-96.1)$. Conclusions: Values were established for newborns at the height of the City of Mexico, with which we can suggest the application of a safe cardiac sieve with the guidelines of the AAP in our environment.

Keywords: Saturation in new born, altitude in Mexico City, cardiac sieve.

miento, de estos casos, alrededor de $25 \%$ se consideran defectos graves, los cuales se definen como las CC que requieren de tratamientos quirúrgicos o cateterismos cardiacos en el primer año de vida. En México de 3 a 4\% de las

\footnotetext{
* Residente de Neonatología. Facultad Mexicana de Medicina de la Universidad La Salle. México.

‡ Cardiología Pediátrica, Departamento de Pediatría.

$\S$ Jefe del Departamento de Pediatría y Neonatología.

॥ Jefe de Cunero Fisiológico.

^ Instituto Nacional de Salud Pública y Departamento de Pediatría.
}

Hospital Español de México. Ciudad de México.

Correspondencia:

Dra. Corinne Michelle Lozano Duau

Correo electrónico: corinnemld89@hotmail.com

Aceptado: 02-10-2019.

Abreviaturas:

AAP $=$ American Academy of Pediatrics.

$\mathrm{RN}=$ Recién nacidos.

$\mathrm{CC}=$ Cardiopatías congénitas.

$\mathrm{TC}=$ Tamiz cardiológico.

SDG $=$ Semanas de gestación.

$\mathrm{SpO}_{2}=$ Saturación de oxígeno.

$\mathrm{m}=$ Metros

www.medigraphic.com/actamedica

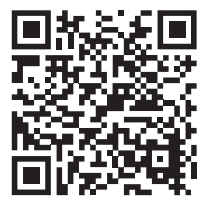


malformaciones congénitas corresponden a cardiopatías. Hoy en día se acepta una incidencia de 0.8-1.4\%, la cual ha ido en aumento, probablemente por la mayor precisión diagnóstica. ${ }^{1-3} \mathrm{El}$ uso del tamiz cardiológico (TC) para la detección de CC en recién nacidos (RN) se estableció en el año 2011 por la AAP, posterior a estudios realizados entre 2004 y 2007 en los cuales se comprobaba que existía un beneficio de este tipo de estudio para la detección de cardiopatías en comparación con el uso únicamente de la exploración física. ${ }^{4}$ A partir de estos hallazgos en ocho estados de Estados Unidos se estableció un plan de tamizaje. Estos estados tuvieron 33\% de reducción en la mortalidad infantil temprana debido a las CC en comparación con la mortalidad en otros estados donde no se aplicó este estudio, posteriormente, el TC se implementó en las unidades de pediatría de varios países con éxito. ${ }^{5}$

En los últimos años se ha cuestionado si la aplicación del tamiz se debe modificar en sus valores de referencia, en relación con la altitud sobre el nivel del mar del lugar donde se lleva a cabo el estudio. Por ello llevamos a cabo este estudio en el cual nuestro objetivo fue describir la saturación pre y postductal realizada mediante el tamiz cardiaco recomendado por la AAP de todos los RN ingresados al cunero fisiológico de un hospital privado de la Cuidad de México, durante el periodo de marzo a agosto del 2018, a una altitud de 2,640 metros sobre el nivel del mar.

\section{MATERIAL Y MÉTODOS}

Se realizó un estudio descriptivo de tipo transversal en el cunero fisiológico del Hospital Español de la Ciudad de
México de marzo a agosto de 2018. Se incluyeron todos los recién nacidos con más de 24 horas de vida que se encontraban en el cunero fisiológico, sin importar el uso o no de oxígeno en el periodo de transición. Los criterios de exclusión fueron patología pulmonar, infecciosa, gastrointestinal, cardiopatía, error innato del metabolismo, síndrome de respuesta inflamatoria sistémica, cianosis central o periférica, o cualquier otra situación que dejara al RN fuera del rubro de "clínicamente sano".

\section{Tamiz cardiológico}

Las determinaciones se realizaron con un oxímetro de pulso (Phillips, monitor multiparamétrico de $\mathrm{CO}_{2} / \mathrm{Pl} / \mathrm{Temp} . / \mathrm{SpO}_{2}$, IntelliVue MP5. USA), el algoritmo utilizado fue el de la AAP (Figura 1), el cual establece que debe realizarse a las 24-48 horas del nacimiento, el paciente debe estar con aire ambiente en el momento de la toma de saturación, calmado, y eutérmico. ${ }^{6-8} \mathrm{El}$ muestreo fue sistemático, incluyendo a todos los pacientes conforme fueron ingresando al servicio del hospital. La toma de oximetría se realizó en dos sitios: mano derecha (preductal) y cualquiera de los pies (postductal), con tres resultados posibles:

Positivo inmediato: si la saturación es menor de 90\% en la mano derecha o alguno de los pies.

Positivo: si la saturación es de 90-94\% en la mano derecha y alguno de los pies, o hay una diferencia de saturación mayor de 3\% entre medición preductal y postductal.

Negativo: si la saturación en ambas extremidades es mayor de $94 \%$ o la diferencia es menor de $3 \%$.

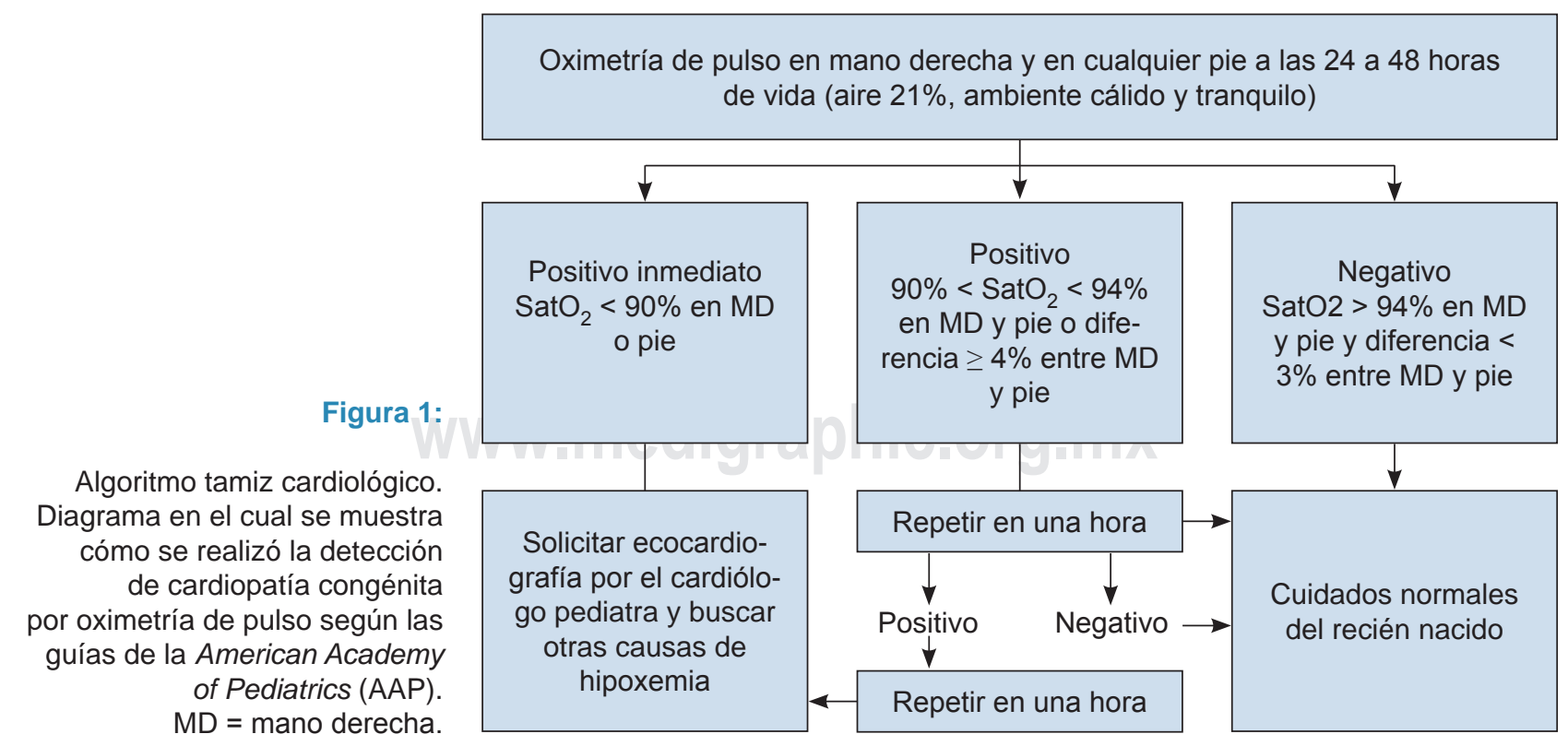


Tabla 1: Características de la población

estudiada; se muestra edad gestacional, peso al nacimiento y el uso de oxígeno ( $\mathrm{N}=292)$.

\begin{tabular}{lc} 
Parámetro & Valor obtenido \\
\hline Edad gestacional & \\
Media \pm DE (SDG) & $38 \pm 1.4$ \\
Mínimo-máximo (SDG) & $29.4-42$ \\
Pretérmino tardío (\%) & 12.30 \\
Término (\%) & 87.70 \\
Peso al nacimiento (g) & \\
$\quad$ Media \pm DE & $2,940 \pm 410$ \\
Mínimo-máximo & $2,000-4,330$ \\
Bajo peso (\%) & \\
Sí & 15.80 \\
No & 84.20 \\
Uso de oxígeno (\%) & \\
Sí & 5.50 \\
No & 94.50
\end{tabular}

DE = desviación estándar, $\mathrm{SDG}$ = semanas de gestación, $\mathrm{g}$ = gramos .

Cuando la prueba es dudosa o es positiva, se repite el estudio en dos ocasiones más, con diferencia de una hora cada una. Si la saturación de oxígeno continúa siendo entre 90 y $95 \%$ y la diferencia entre las mediciones del pie y de la mano es mayor de $3 \%$, el tamiz se considera anormal, en cuyo caso el niño debe ser referido al servicio de cardiología pediátrica para su evaluación urgente con un ecocardiograma. Si el resultado es positivo inmediato se solicita la valoración del servicio mencionado.

\section{RESULTADOS}

Realizamos un total de 292 estudios en RN sanos, con una media de edad gestacional de $38 \pm 1.4$ SDG, teniendo una máxima de 42 SDG y una mínima de 29.4 SDG, con un predominio de $\mathrm{RN}$ de término $(87.7 \%)$, en cuanto al peso al nacimiento la media fue de 2,940 gramos, con un peso máximo de 4.330 gramos y un mínimo de 2,000 gramos. Se usó oxígeno sólo en 5.5\% de los RN (16 pacientes) de forma transicional al nacimiento, el cual se retiró posterior a las seis horas de vida, estos RN no tuvieron complicaciones postparto y al momento del estudio ya no tenían oxígeno. $46 \mathrm{RN}$ presentaron peso bajo al nacer; sin embargo, esto no se asoció a menor saturación ni pre ni postductal (Tabla 1).

Del total de los pacientes estudiados $(n=292)$ sólo en tres pacientes, requerimos realizar una segunda medición debido a que el resultado fue positivo, las saturaciones preductales reportadas fueron entre $90-94 \%$, por lo que se repitieron una hora posterior dando un valor $>94 \%$, por lo tanto no tuvimos pacientes a los cuales se les solicitara la valoración por cardiología, finalmente no tuvimos ningún reingreso de estos RN por alguna patología cardiaca o pulmonar (Tabla 3).

\section{DISCUSIÓN}

Los niveles de oxigenación $\left(\mathrm{SpO}_{2}\right)$ en el $\mathrm{RN}$ han sido un parámetro utilizado para evaluar la adaptación del recién nacido al ambiente. Sin embargo, existen pocas investigaciones que midan la $\mathrm{SpO}_{2}$ por oximetría de pulso en el $\mathrm{RN}$ de término y pretérmino tardío clínicamente sano a una altitud similar a la de la Ciudad de México (2,640 metros sobre el nivel del mar), esta falta de valores de referencia, sobre todo de mínimos aceptables a esta altitud, hace que cuando se requiera, se manejen valores obtenidos al nivel de mar, de ahí la necesidad de disponer de ellos para precisar mejor el manejo de RN. ${ }^{9,10}$

La $\mathrm{SpO}_{2}$, medida a través de oximetría de pulso por reflectancia al nacimiento es de $60 \%$ y aumenta gradualmente durante los primeros 10 minutos de vida hasta llegar a $95 \%$, Orozco y colaboradores reportaron diferencias entre la saturación al nacimiento y a las 48 horas, y si se modificaba con el uso de oxígeno en la reanimación en 146 neonatos, concluyendo una $\mathrm{SpO}_{2}$ media al nacimiento pre y postductal de $92 \%$ y a las 48 horas una media pre y postductal de $96 \%$, sin encontrar diferencias significativas entre el uso o no de oxígeno. ${ }^{10}$

A nivel de la Ciudad de México se han realizado dos estudios por medio de gasometría arterial (punción arterial): uno en $\mathrm{RN}$ sanos de término en los primeros días de vida extrauterina, encontrando que la saturación de oxígeno central osciló entre 86 y 96.5\%, con un promedio de 92.3 .

Tabla 2: Valores de saturación periférica de oxígeno por oximetría de pulso de forma preductal y postductal, con valores mínimos y máximos, así como las medias.
Valores de saturación

por oximetría de pulso

Oximetría preductal Media \pm DE Mínimo-máximo

Oximetría postductal

Media \pm DE

Mínimo-máximo
DE = desviación estándar.
Valor obtenido

(\%)
$95.5 \pm 2.4$

90.0-100

$95.8 \pm 2.4$

90.0-100 


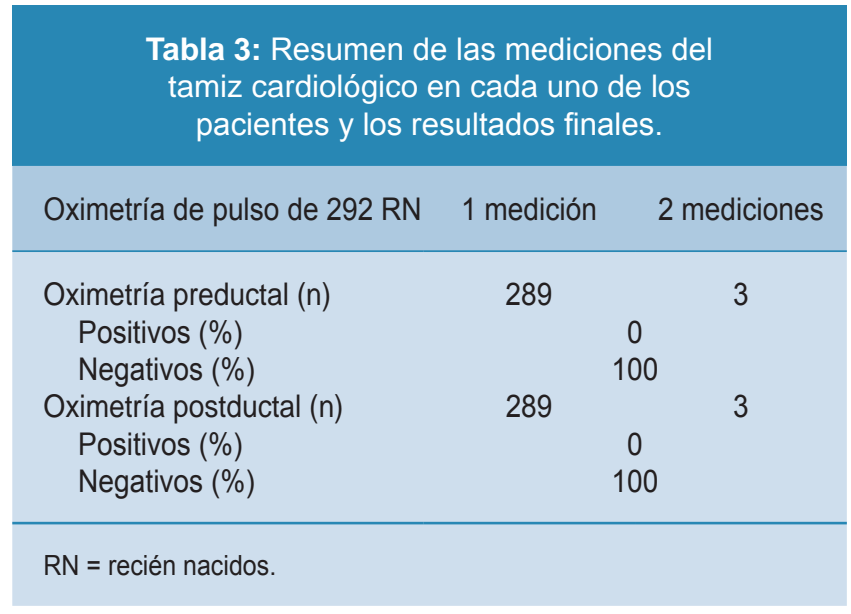

Este estudio fue realizado en $47 \mathrm{RN}$, tanto de término como pretérmino, cuatro con hiperbilirrubinemia, dos con antecedente de síndrome de distrés respiratorio leve, uno con enterocolitis necrosante estadio IIA, uno con conjuntivitis y otro con síndrome de Down sin cardiopatía; el resto era sano. La gasometría mostró un mínimo de $83.4 \%$ y un máximo de $95.2 \%$, con un promedio de $90.5 \pm 2.7 \%$; en ese estudio dos casos se desaturaron probablemente por llanto sostenido, los demás estaban por arriba de 50 $\mathrm{mmHg}$ de $\mathrm{PaO}_{2}$ y con saturaciones por arriba de la más baja mencionada con anterioridad. ${ }^{11}$

No se obtuvo diferencia significativa en las saturaciones pre y postductales con el uso de oxígeno en el periodo de transición, ni hubo diferencia entre recién nacidos de término y de pretérmino tardío. Quisimos determinar dichas saturaciones y compararlas con las establecidas a nivel del mar, y saber si se tendría que hacer una modificación en el algoritmo de tamizaje cardiaco ajustado a nuestra altitud con el fin de diagnosticar de manera correcta cardiopatías detectables por dicho tamiz. No se encontró variabilidad significativa en la saturación, por lo que se puede seguir de forma segura el lineamiento del algoritmo propuesto por la Academia Americana de Pediatría sin cambiar los valores de saturación pre y postductal.

\section{Limitaciones del estudio}

Nuestro estudio cuenta con algunas limitaciones que deben ser mencionadas. La primera es que el tamaño de nuestra muestra es reducido y nos beneficiaríamos de una muestra mayor. Sin embargo, no tenemos motivos para pensar que la saturación difiera mucho. La segunda limitación es que sólo contamos con un método para medir la saturación. Sin embargo, el oxímetro de pulso se considera una prueba sensible para estas mediciones.

\section{CONCLUSIONES}

Los $\mathrm{RN}$ con pruebas anormales de saturación de oxígeno no necesariamente tienen una CC, existen otras patologías que también pueden cursar con hipoxemia, por ejemplo, sufrimiento respiratorio agudo e hipertensión pulmonar por persistencia del patrón pulmonar fetal, entre otras. También se ha observado que puede haber pequeñas variaciones en el resultado de la saturación de oxígeno cuando el tamiz se realiza en niños dormidos, por lo que es recomendable que se efectúe mientras están despiertos. Por otro lado, los padres y los médicos deben saber que una prueba de saturación normal no descarta la presencia de otras cardiopatíass congénitas que no cursan con hipoxemia. Finalmente, es importante mencionar que en pacientes pretérmino no tenemos muchas referencias sobre valores normales de saturación, por lo que manejamos en la mayoría de las veces valores obtenidos a nivel del mar de recién nacidos de término; de ahí la necesidad de realizar más estudios en este grupo de edad.

Sobre el uso del saturómetro Phillips, monitor multiparamétrico de $\mathrm{CO}_{2} / \mathrm{PI} / \mathrm{Temp}$./SpO $\mathrm{Sp}_{2}$ IntelliVue MP5 se puede destacar que se observaron menos falsos positivos, versus el uso rutinario para tamizaje cardiaco del equipo Massimo, por lo que se podría recomendar para uso rutinario en un futuro. ${ }^{12}$

\section{REFERENCIAS}

1. Tennant PW, Pearce MS, Bythell M, Rankin J. 20-year survival of children born with congenital anomalies: a population-based study. Lancet. 2010; 375 (9715): 649-656. doi: 10.1016/S01406736(09)61922-X.

2. Bird TM, Hobbs CA, Cleves MA, Tilford JM, Robbins JM. National rates of birth defects among hospitalized newborns. Birth Defects Res Part A Clin Mol Teratol. 2006; 76 (11): 762-769. doi: 10.1002/ bdra.20323.

3. Canfield MA, Honein MA, Yuskiv $\mathrm{N}$ et al. National estimates and race/ethnic-specific variation of selected birth defects in the United States, 1999-2001. Birth Defects Res Part A Clin Mol Teratol. 2006; 76 (11): 747-756. doi: 10.1002/bdra.20294.

4. Garg LF, Van Naarden Braun K, Knapp MM et al. Results from the new jersey statewide critical congenital heart defects screening program. Pediatrics. 2013; 132 (2): e314-e323. doi: 10.1542/ peds.2013-0269.

5. Abouk R, Grosse SD, Ailes EC, Oster ME. Association of US state implementation of newborn screening policies for critical congenital heart disease with early infant cardiac deaths. JAMA. 2017; 318 (21): 2111-2118. doi: 10.1001/jama.2017.17627.

6. Cullen BPJ, Guzmán CB. Tamiz de cardiopatías congénitas críticas. Recomendaciones actuales. Acta Med. 2014; 12 (1): 24-29.

7. Marino BS, Lipkin PH, Newburger JW et al. Neurodevelopmental outcomes in children with congenital heart disease: evaluation and management. Circulation. 2012; 126 (9): 1143-1172. doi: 10.1161/ CIR.0b013e318265ee8a

8. Kemper AR, Mahle WT, Martin GR et al. Strategies for implementing screening for critical congenital heart disease. 
Pediatrics. 2011; 128 (5): e1259-e1267. doi: 10.1542/ peds.2011-1317.

9. Koestenberger M, Nagel B, Ravekes W et al. Systolic right ventricular function in preterm and term neonates: reference values of the tricuspid annular plane systolic excursion (TAPSE) in 258 patients and calculation of z-score values. Neonatology. 2011; 100 (1): 85-92. doi: 10.1159/000322006.

10. Orozco Gutiérrez A, Pereira Díaz A, Marcelo Orozco S. Diferencias entre la saturación de oxígeno al nacer y las 48 horas en neonatos a término reanimados con y sin oxígeno. Acta Med. 2017; 15 (4): 264-268.

11. Tapia-Rombo CA, Rosales-Cervantes MGI, Saucedo-Zavala VJ et al. Saturación periférica de oxígeno por oximetría de pulso en recién nacidos clínicamente sanos a la altitud de la Ciudad de México (2240 m). Gac Med Mex. 2008; 144 (3): 207-212.

12. Peña-Juárez RA, Chávez-Sáenz JA, García-Canales A et al. Comparación de oxímetros para detección de cardiopatías congénitas críticas. Arch Cardiol Mex. 2019; 89 (2): 172-180. 